\title{
EI DERECHO LABORAL ANTE EL RETO DE LA ECONOMÍA COMPARIDA: APPS, SMARTPHONES Y TRABAJO HUMANO.
}

\section{THE LABOR LAW TO THE CHALLENGE OF ECONOMICS COMPARIDA : APPS, SMARTPHONES AND HUMAN LABOR.}

Gustavo Gauthier1

Resumen: Los desafíos que la historia había reservado a la ley del Trabajo , presenta hoy un nuevo episodio como resultado de un nuevo emerge forma de comercio de bienes y servicios que se ha registrado un crecimiento exponencial en el contexto de lo que se ha llamado " el intercambio o colaboración economía ". El primer reto es ahora para entender el alcance del nuevo fenómeno, y luego analizar si las categorías y herramientas tradicionales de la disciplina, son adecuados o capaz de decodificar la nueva forma de servicio que se produce con las nuevas empresas de la "economía del compartir"

Palavras-chave: economía compartida, aplicaciones, trabajo humano.

\begin{abstract}
The challenges that history had reserved to the Labor law, presents today a new episode as a result of a new emerges form of trade in goods and services that has registered an exponential growth in the context of what has been called "sharing or collaborative economy". The first challenge is now to understand the scope of the new phenomenon, and then analyze whether the categories and traditional tools of the discipline, are suitable or able to decode the new form of service that occurs with new ventures of the "sharing economy".
\end{abstract}

Keywords: shared economy, applications, human labor.

1 Doutor em Direito e Ciências Sociais pela Universidad de la República, Montevideo, Uruguai. 


\section{LA ECONOMÍA COMPARTIDA.}

El concepto de consumo colaborativo comenzó a popularizarse en 2010 con la publicación del libro "What's Mine Is Yours: The Rise of Collaborative Consumption". Rachel Botsman, co-autora del mismo, ofreció una charla sobre consumo colaborativo en la conferencia TEDxSydney en mayo de 2010, en la que expuso cómo el acceso a bienes y servicios puede ser de relevancia prioritaria sin ser necesaria la propiedad de los mismos. ${ }^{1}$

Más allá del boom tecnológico de los últimos años debido al auge de las redes sociales y la salida a bolsa de Fecebook, Twitter y Linkedin, hoy en día los ojos de Silicon Valley, complejo que reúne a las mayores corporaciones de tecnología del mundo, apuntan hacia el modelo de negocio de la economía compartida, definida por Tim Bradshaw del Financial Time como "aquella que facilita el intercambio directo de bienes y servicios entre los particulares". 2

En otros casos, como sinónimo de economía compartida, se hace mención a la "economía colaborativa", para referir a todas aquellas plataformas y negocios creados alrededor del concepto del consumo colaborativo entre ciudadanos, ya sea ofrecido por una entidad pública o privada o incluso entre pares.

En el fondo del fenómeno, se encuentra un cambio de concepto entre el poseer un determinado bien y el acceder al mismo. Ahora ya no importa tanto el poseer un determinado producto o servicio, sino lo que importa es el poder acceder a su disfrute, y basándose en este concepto muchos emprendedores han creado sus propias plataformas de consumo colaborativo

Si bien es cierto que la economía colaborativa o compartida no es un fenómeno que haya surgido en los últimos años, la economía compartida siempre ha estado ahí, lo que está sucediendo actualmente es una especie de aceleración inimaginable poco tiempo atrás.

La aceleración de la "economía compartida" reconoce una serie de factores que en los últimos años han propiciado la proliferación de este fenómeno; entre ellos, los más importantes y que todo el mundo coincide son:

\footnotetext{
${ }^{1}$ https://es.wikipedia.org/wiki/Consumo_colaborativo

2 www.elfinanciero.com.mx/opinion/la-economia-compartida.html
} 
- La crisis económica: la mayoría de los ciudadanos han visto como sus capacidades económicas, han disminuido por la situación económica actual, por lo que la opción de poseer y mantener ciertos productos se hace imposible e inviable.

- La revolución digital y la tecnología: los smarthpones, tablets y portátiles de última generación han propiciado la generalización del acceso a internet, pero también el concepto de siempre "online", pudiendo acceder a internet casi desde cualquier lugar y en cualquier momento. Este hecho ha supuesto un impulso definitivo en la creación de múltiples plataformas online que ofrecen economía colaborativa pero también en que tengan éxito.

- La conciencia medioambiental: el consumo compartido implica una reducción del impacto medioambiental, un sentimiento bastante arraigado en la sociedad en los últimos años.

De acuerdo a Forbes, la economía compartida crecerá en 2016 un 25\%, con 3.5 billones de dólares estimados en ingresos entrando directo a los bolsillos de la gente, más los ingresos sustanciales que reciben cada una de estas compañías por enlazar a compradores y vendedores. ${ }^{3}$

La masificación creciente de los denominados teléfonos inteligentes o smartphones y el desarrollo de las más diversas aplicaciones o apps, constituyen la plataforma que hace posible actualmente un crecimiento exponencial de la economía compartida. Si bien las aplicaciones están presentes en los teléfonos desde hace tiempo (calculadoras, alarmas, agendas, etc.), los primeros teléfonos inteligentes contaban con pantallas reducidas y muchas veces no táctiles que no permitían ciertas funcionalidades imprescindibles para el desarrollo de las aplicaciones actuales.

En esencia, una aplicación no deja de ser un software. Para entender un poco mejor el concepto, podemos decir que las aplicaciones son para los móviles lo que los programas son para los ordenadores de escritorio.

Una aplicación informática es un tipo de software que permite al usuario realizar uno o más tipos de trabajo y de vincularse con la oferta y la demanda de servicios.

El desarrollo de la tecnología y la revolución digital han llevado a la creación de una auténtica revolución, de la que han surgido miles de negocios basándose en el concepto de "Acceder" a un producto sin poseerlo:

\footnotetext{
${ }^{3}$ www.forbes.com.mx/la-verdad-sobre-el-share-economy
} 
- Ya no es necesario poseer un CD para poder escuchar una canción, para ello existen Spotify o GrooveShark.

- Ya no es necesario acudir a un banco para obtener un préstamo, existen plataformas como Comunitae o Zopa, que ponen en contacto personas que tienen exceso de dinero con personas que lo necesitan en ese momento y ambas obtienen un beneficio.

- Otras plataformas, como tuplaza.es, ponen en contacto personas que viven o trabajan en el mismo barrio para compartir el uso de plazas de garaje y así compartir también el gasto que conllevan.

Pero también existen aplicaciones que tienen como finalidad vincular la oferta y la demanda de servicios:

- Airbnb, pone en contacto personas que buscan alojamiento para vacaciones, escapadas, viajes de negocio, con personas que tienen en sus casas o alojamientos sin uso y que están dispuestas a recibir a viajeros.

Esta empresa, maneja una oferta de más de 800.000 viviendas en 190 países (como comparativo: el grupo Hilton, segunda cadena hotelera mundial, tiene 680.000 habitaciones en 4100 hoteles). En su primer año de operaciones (2009), Airbnb registró 21.000 pernoctaciones, llegando dos años después a casi un millón; en la actualidad registra un millón de pernoctaciones mensuales.

-Uber, es una aplicación que opera servicios de transportación privada en algunas de las ciudades más importantes del mundo, desde Los Ángeles hasta Hong Kong a un precio similar o más barato al de un taxi. Uber ofrece un servicio similar al de un chofer privado, con Wi-Fi en la unidad, agua de cortesía y atención personalizada. Además, permite rastrear por GPS a la unidad, se paga con tarjeta de crédito desde el celular, se puede dividir la tarifa entre los integrantes vía electrónica y aparecen los datos de contacto del chofer para mayor seguridad y en caso de algún imprevisto.

A diciembre de 2014, Uber realizaba un promedio de 2.000.000 de viajes diarios, la mitad solo en algunas ciudades de China, y Bloomberg ha estimado el valor de la compañía en 40.000 millones de dólares.

\section{2 ¿POR QUÉ LA ECONOMÍA COMPARTIDA REPRESENTA UN NUEVO DESAFÍO PARA EL DERECHO DEL TRABAJO?}


El tipo de negocio que se plantea en la economía compartida, por lo general se sitúa más allá o fuera del alcance de las regulaciones estatales bajo las cuales de ordinario operan las empresas tradicionales. Ello por cuanto tanto el intercambio de bienes como el de servicios, independientemente de que se trate de transacciones que poseen un determinado valor económico para las partes involucradas, desafía las reglas fiscales, las reglas de las activiades reguladas por el Estado, las reglas de la responsabilidad de las personas y/o empresas intervinientes, las normas de cobertura de los distintos riesgos involucrados a través de pólizas de seguros, etc. La novedad y peculiaridad de esta nueva economía hace muchas veces difícil o imposible de aplicar reglas o normas de la más diversa índole concebidas para otro tipo de realiades o negocios.

Las reglas del Derecho del trabajo no escapan a esta realidad, a punto de considerar que las personas que prestan servicios para este tipo de aplicaciones en principio no son empleados de las empresas propietarias de las plataformas, sino que son considerados contratistas independientes o particulares autónomos.

El desafío se centra en determinar si realmente se trata de contratistas o trabajadores autónomos en función de la peculiar forma en que los servicios se prestan o si, por el contrario, se trata de trabajadores dependendientes que a primera vista aparecen, o los hacen aparecer, como socios o contratistas independientes.

En esta tarea, necesariamente se pondrá a prueba el arsenal de inidicios que tradicionalmente se reconocen como válidos en el Derecho del trabajo para determinar la existencia de una relación de empleo, a efectos de establecer su validez en el marco de la economía compartida o la necesidad de plantear otros indicios o herramientas nuevas suscpetibles de comprender y regular el nuevo fenómeno.

\section{EL CASO "UBER"}

A efectos de profundizar en las características del tipo de intercambio que se produce en la economía compartida, resulta pertinente detenerse en el análisis del caso de la empresa Uber, que como se adelantara opera servicios de transportación privada en algunas de las ciudades más importantes del mundo, y que resulta particularmente interesante por tratarse de la empresa líder a nivel global en la prestación estos servicios y que es similar a otras pertenecientes al mismo rubro como Easy Taxi, y también porque comienzan a aparecer las primeras sentencias en Estados Unidos donde se analiza precisamente la naturaleza del vínculo entre Uber y sus choferes. 
Asimismo, no es posible soslayar que el ingreso de Uber en los países donde ha pretendido comenzar a operar no ha sido y no está siendo fácil o pacífica, fundamentalmente debido a la oposición que suele tener de parte de los sindicatos de taxistas que ven amenazado su trabajo por la irrupción de la nueva modalidad de transporte.

Alemania por ejemplo prohibió la actividad de Uber, la empresa tiene procesos judiciales abiertos en Holanda, Brasil y España y el 3 de julio de 2015 anunció su retiro de Francia ante la presión del gobierno y la detención de sus ejecutivos.

Por otra parte, México ha sido el primer país de América latina que ha optado recientemente - 17 de julio de 2015- por regular el servicio de choferes de Uber y otras compañías, creando un impuesto de $1,5 \%$ del costo de cada viaje.

En Uruguay, el 30 de junio de 2015, la Comisión de Promoción y Defensa de la Competencia, creada por Ley No. 18.159, en una investigación de oficio sobre el mercado relevante de servicios de intermediación para el transporte de taxímetro en Montevideo, tanto telefónico como por internet u otros, determinó el cese inmediato de la conducta de prohibición e imposición de sanciones a todos los afiliados al Centro de Propietarios de Automóviles con Taxímetro del Uruguay por utilizar otros medios de pedidos de taxis (EasyTaxi) diferentes a los desarrollados por dicha gremial, quedando permitido el libre uso de cualquiera de ellos. Ello podría representar un antecedente favorable para un eventual ingreso de Uber a operar en Uruguay, extremo que aún no se ha verificado.

En primer lugar corresponde detenerse en las características del vínculo de Uber, con los usuarios del servicio, esto es, con las personas que debiendo trasladarse de un lugar a otro - por falta de un vehículo propio o por preferir no utilizar el mismo- requiren el servicio de transporte personalizado de Uber, antes que tomar un taxi:

- La app de Uber contiene información y constituye un medio de obtener servicios de transporte ofrecidos por terceros que son proveedores de transporte, conductores $\mathrm{u}$ operadores de vehículos (el Proveedor de transporte)

- Uber por sí misma no presta directamente servicios de transporte y no se asume como transportista.

- El Proveedor del Transporte es quien decide ofrecer servicios de transporte, que se pueden solicitar mediante el uso de la app.

- Uber solo actúa como intermediario entre el usuario y el Proveedor del Transporte. 
- La prestación de los servicios de transporte del Proveedor del Transporte está sujeta al acuerdo entre el usuario y el Proveedor del Transporte. Uber no es parte de este acuerdo.

- Uber cobra por los servicios de transporte prestados por el Proveedor del Transporte en nombre del mismo.

- La calidad de los servicios de transporte solicitados a través del uso de la aplicación será plenamente responsabilidad del Proveedor del Transporte, quien en última instancia será quien preste los servicios de transporte.

- Bajo ninguna circunstancia Uber acepta ninguna responsabilidad en relación con o derivada de los servicios de transporte prestados por el Proveedor del Transporte ni por ningún acto, acción, comportamiento, conducta o negligencia por parte del Proveedor del Transporte. Todas las reclamaciones sobre los servicios de transporte prestados por el Proveedor del Transporte se deben remitir al Proveedor del Transporte.

En segundo término, corresponde analizar, hasta done ello es posible, las características del vínculo de Uber con los choferes o "Proveedores de Transporte" como se los denomina y que no son otros que personas que adquieren o poseen un vehículo y se registran en la plataforma de la empresa a efectos de ofrecer transporte personalizado:

- Al enviar la solicitud de ingreso, el chofer autoriza a Uber, y a su contratista de seguridad, para la consulta y obtención de información de cualquier fuente legítima y legal, de diferentes bases de datos de autoridades competentes a nivel nacional. Entre ellas, entidades del Sector Financiero, Judicial y Policial, entre otras. De igual forma, acepta la verificación de los antecedentes y referencias personales o cualquier otra información personal.

- Las tarifas de los servicios son sugeridas por Uber.

- Uber entrega a cada chofer un smartphone con la aplicación instalada.

- El vehículo y todos los gastos asociados están a cargo de los propietarios de los mismos.

- Uber cobra el precio del viaje directamente al usuario, retiene una comisión de entre 20\%, y le paga el saldo restante al Proveedor de Transporte o chofer.

- Uber sugiere a los conductores vestir profesionalmente o escuchar jazz suave.

- Uber puede cancelar el contrato con el conductor que no sigue las sugerencias o en virtud de quejas que los usuarios pueden subir a la plataforma. 
Como se puede apreciar, existen un sinnúmero de indicios que podrían denotar la existencia de una relación independiente entre Uber y los choferes, como también muchos otros que resultan más propios de una relación de empleo.

Es por ello que resulta particularmente interesante el análisis contenido en los primero fallos que están apareciendo en el Estado de California y que los especialistas en Derecho Laboral de Estados Unidos están observando cuidadosamente.

\section{RECIENTE JURISPRUDENCIA DEL TRIBUNAL DEL DISTRITO NORTE DE CALIFORNIA.}

En una sentencia de 11 de marzo de 2015, dictada por la Corte del Distrito Norte de California en el caso "Douglas O'Connor v. Uber Technologies Inc.”, se abordó el reclamo de conductores de California que reclaman ser considerados empleados y no contratistas independientes. ${ }^{4}$

El antecedente jurisprudencial inmediato, es de una Corte Federal de 2014, donde se reconoció el carácter de empleados a conductores de camiones de FedEx, que se vinculaban con la empresa de forma similar a como se vinculan los choferes de Uber. ${ }^{5}$

Siendo que bajo la ley de California existe una presunción de que un proveedor de servicios es empleado a menos que se demuestre lo contrario, en la referida sentencia el Juez sostuvo que:

- En materia de control, el Tribunal Supremo ha destacado que la pregunta pertinente no es cuánto contralor ejerce el arrendatario, sino la cantidad de control que el arrendatario se reserva el derecho a ejercer.

- Aún más fundamental, es que los conductores realizan un servicio para Uber porque simplemente no habría una entidad viable sin sus conductores.

- Los ingresos de Uber no dependen de la distribución de su software, sino de la generación de viajes por sus conductores.

\footnotetext{
${ }^{4}$ Case3:13-cv-03826-EMC Document251 Filed03/11/15
}

${ }^{5}$ Alexander, 765 F.3d at 985 
- Los pasajeros pagan directamente a Uber la totalidad del importe de la tarifa, luego Uber paga a sus conductores el $80 \%$ de la tarifa que cobra el chofer, quedándose con el $20 \%$ restante.

- En pocas palabras, los contratos confirman que Uber únicamente hace dinero si sus conductores transportan pasajeros.

- Como más indicios de su papel como una empresa de transporte en lugar de un proveedor de software, Uber ejerce un control sustancial sobre la calificación y selección de sus conductores, que previo a su ingreso deben someterse a un proceso que incluye una revisión de antecedentes, un cuidadoso examen de conocimiento, la inspección del vehículo y una entrevista personal.

- Los conductores de Uber proporcionan un "servicio indispensable" para Uber y la empresa "no podría sobrevivir sin ellos" de lo que podría sin una aplicación de teléfono inteligente; o dicho más coloquialmente, Uber no podría sostener su slogan "Todo el mundo con su chofer privado", sin los conductores.

- Los demandantes citan numerosos documentos, que instruyen a los conductores, entre otras cosas: "Asegúrese de que está vestido profesionalmente"; "enviar al cliente un mensaje de texto 1-2 minutos antes del lugar de recogida ("Esto es muy importante")"; "Asegúrese de que la radio está apagada o en jazz suave o NPR" y "asegúrese de abrir la puerta para que su cliente aborde el vehículo", como Uber subraya, "son los pequeños detalles que hacen un excelente viaje".

- Los conductores de Uber, son monitoreados por los clientes (para beneficio de Uber, ya que ésta utiliza la calificación de clientes para tomar decisiones con respecto a los conductores), independientemente que durante todos y cada viaje la aplicación de Uber proporciona datos para monitorear constantemente algunos aspectos de la conducta de un conductor.

- Este nivel de vigilancia, donde los conductores son potencialmente observables en todo momento, podría decirse que da a Uber una tremenda cantidad de control sobre la "manera y los medios" de la actuación de sus pilotos. Si el monitoreo trimestral en el caso "Alexander" era lo suficientemente generalizada para pesar a favor de que los conductores de FedEx eran empleados, un jurado razonable podría concluir que la supervisión del rendimiento más persistente de Uber, pesa de manera similar en favor de encontrar que los conductores son empleados de Uber bajo la ley de California. 
- Sin duda, una serie de factores secundarios (por ejemplo, los conductores utilizan su propio vehículo, puede emplear otros conductores para conducir en su nombre y firmaron un acuerdo en el que no hay relación de empleo), apoyan una clasificación de contratista independiente.

- Pero incluso en cuanto a estos factores, su significado es ambiguo. Por ejemplo, el hecho de que los controladores proporcionan sus propios vehículos y por lo tanto invierten un importante capital es un factor importante a favor de una relación de contratista independiente pero este hecho por sí solo no es determinante. En "Alexander v FedEx", se encontró relación de empleo a pesar de que los conductores siempre manejaban sus propios vehículos.

- Por otra parte, existe un factor calificado, como el hecho de que Uber suministra la herramienta fundamental de la empresa: un teléfono inteligente con la aplicación de Uber.

En base a estas consideraciones, los actores evitaron que su demanda fuera rechazada en una primera instancia de calificación de puro derecho, estando en plena instrucción el juicio en la siguiente instancia.

No obstante y a propósito del desafío que para el Derecho del trabajo representan estas nuevas modalidades de prestación de servicios personales, resultan particularmente interesantes las reflexiones que a modo de conclusión efectúa el Juez, Edward M. Chen, en la sentencia y que perfectamemte podrían hacer las veces de conclusiones del presente trabajo:

* La prueba tradicional de la relación de empleo, que se desarrolló bajo un modelo económico muy diferente al de la nueva "economía compartida" y al modelo de negocio de Uber, crea desafíos significativos.

* Podría decirse que muchos de los factores en esa prueba aparecerá anticuado en este contexto.

* Otros factores que podrían posiblemente ser un reflejo de las realidades económicas actuales (como la proporción de los ingresos generados y compartidos por las partes respectivas, su poder de negociación relativo, y la gama de alternativas disponibles para cada uno), no están comprendidos expresamente por el modelo tradicional.

* Es posible que los tribunales de apelación o la legislatura puedan refinar o revisar la prueba indiciaria en el contexto de la nueva economía. 
* Es concebible que el legislador promulgue reglas particulares a la nueva llamada "economía compartida."

* Hasta entonces, esta Corte tiene la tarea de aplicar la prueba multifactorial tradicional a los hechos en cuestión. Por las razones expuestas anteriormente, aparte de la conclusión preliminar de que los conductores Uber son empleados presuntivos, los indicios tradicionales no producen un resultado inequívoco. 